\title{
Psychological interventions to improve glycaemic control in patients with type 1 diabetes: systematic review and meta-analysis of randomised controlled trials
}

\author{
Kirsty Winkley, Sabine Landau, Ivan Eisler, Khalida Ismail
}

\begin{abstract}
Objective To determine whether psychological interventions have any effect on glycaemic control in people with type 1 diabetes.

Design Systematic review and meta-analysis of psychological therapies to assess their effectiveness in improving glycaemic control in type 1 diabetes.

Data sources Medline, PsycINFO, Embase, and Cochrane central register of controlled trials searched to September 2004. Review methods All included studies were randomised controlled trials in children (including adolescents) or adults with type 1 diabetes that evaluated the effect of a psychological therapy (counselling, cognitive behaviour therapy, family systems therapy, and psychodynamic therapy) on control of diabetes. Data were extracted on sample size, age, duration of diabetes, type of psychological therapy, its mode of delivery, and type of intervention in control group.

Main outcome measures Glycaemic control measured by percentage of glycated haemoglobin and psychological distress. Pooled standardised effect sizes were calculated.

Results 29 trials were eligible for the systematic review and 21 trials for the meta-analysis. In the 10 studies of children and adolescents included in the meta-analysis, the mean percentage of glycated haemoglobin was significantly reduced in those who had received a psychological intervention compared with those in the control group (pooled standardised mean difference $-0.35(95 \%$ confidence interval -0.66 to -0.04$)$, equivalent to a $0.48 \%(0.05 \%$ to $0.91 \%)$ absolute reduction in glycated haemoglobin. In the 11 studies in adults the pooled standardised mean difference was -0.17 ( -0.45 to 0.10$)$, equivalent to $0.22 \%(-0.13 \%$ to $0.56 \%)$ absolute reduction in glycated haemoglobin. Psychological distress was significantly lower in the intervention groups in children and adolescents (pooled standardised effect size $-0.46,-0.83$ to -0.10 ) but not in adults $(-0.25,-0.51$ to 0.01$)$.

Conclusion Psychological treatments can slightly improve glycaemic control in children and adolescents with diabetes but have no effect in adults.
\end{abstract}

\section{Introduction}

People with type 1 diabetes depend on regular insulin injections and on adhering to multiple self care tasks to achieve optimal glycaemic control. Suboptimal glycaemic control ${ }^{12}$ and complications of diabetes are associated with depression, ${ }^{3}$ eating problems, ${ }^{4}$ and fears specific to diabetes. ${ }^{56}$
Considering the rise in the incidence of type 1 diabetes, the limited resources for intensive regimes, ${ }^{7}$ and national guidelines on psychological care in diabetes, ${ }^{8}$ the evidence of effectiveness of psychological therapies in improving diabetes outcomes is poor. Previous reviews have not adequately distinguished between educational and psychological interventions, between type 1 and type 2 diabetes, between randomised and non-randomised trials, or between adults and children when the latter are dependants. ${ }^{19}{ }^{10}$ Children and adolescents have different clinical needs, are assessed by paediatric specialists, and have access to different resources. Education and psychological therapy (not mutually exclusive) are based on different theoretical grounding, training, clinical skills, and are delivered by different specialists. Educational interventions use didactic and enhanced learning methods to improve self management of diabetes by reducing identifiable gaps in knowledge. Psychological therapies use the therapeutic alliance between patient and therapist, in which the patient's problems are understood in terms of emotions, cognitions, and behaviours. Psychological treatments are used widely in medical ${ }^{11}$ and mental health settings ${ }^{12}$ to reduce psychological distress and improve adherence.

We conducted a systematic review and meta-analysis of randomised controlled trials on the effectiveness of psychological therapies in improving glycaemic control in type 1 diabetes. The review was stratified into children and adolescents versus adults with type 1 diabetes to distinguish between the two clinical subgroups. We also assessed whether psychological therapies were effective in reducing psychological distress.

\section{Methods}

\section{Selection of studies}

All studies eligible for inclusion were trials of a psychological intervention, published or unpublished, involving children, adolescents, and adults with a diagnosis of type 1 or insulin dependent diabetes and written in any language.

We categorised type of psychological treatment into four categories: supportive or counselling therapy, ${ }^{13}$ cognitive behaviour therapy, ${ }^{14}$ psychoanalytically informed therapies, ${ }^{15}$ and family systems therapy. ${ }^{16}$ Techniques such as relaxation, activity scheduling, problem solving, goal setting, contract setting, cognitive restructuring, and stress management were categorised as variants of cognitive behaviour therapy, ${ }^{17}$ and motivational interviewing $^{18}$ was categorised under counselling. Delivery of the psy-

The search strategy, QUOROM flow chart, and tables of, and references to, included studies (w1-w34) are on bmj.com. 
chological therapy was defined as individual, group, family (including couple, parents, and multiple families). We included studies where the control group was non-psychological (either usual diabetes care, education, attention control, or waiting list) or less intensive psychological treatment. ${ }^{12}$

Our main outcome measures were long term glycaemic control measured by percentage of glycated haemoglobin, including $\mathrm{HbA}_{1}, \mathrm{HbA}_{1}$, and glycated haemoglobin measurements made by different methods. Our subsidiary outcome was a continuous measure of psychological distress.

\section{Search strategy}

We searched the Cochrane central register of controlled trials on the Cochrane Library (issue 4, 2002), Medline, Embase, and PsycINFO using the following search terms: psychological therapies and mood disorders; diabetes mellitus and clinical trials (see appendix on bmj.com for details of the search strategy). We also hand searched conference proceedings for 1997-2004 (American Diabetes Association, Diabetes UK (formerly British Diabetic Association), European Association for the Study of Diabetes, International Diabetes Federation) under psychological or educational headings. We searched the reference lists of included studies and reviews for additional studies and contacted leading authors of each included trial and experts in the specialty by post or email for additional published or unpublished data.

\section{Data extraction}

Two authors (KI and KW) independently rated abstracts identified by the electronic searches. Inter-rater reliability for trial selection was reported with Cohen's $\kappa .{ }^{19}$ We included any studies that described a controlled trial of a psychological intervention in patients with any form of diabetes. In case of differences of agreement between raters the full original article was retrieved for data extraction.

Hard copies of studies identified at the abstract stage were obtained and the reviewers (KI and $\mathrm{KW}$ ) independently extracted data from each study. Differences over inclusion of studies were resolved through discussions and consensus. Studies written in a foreign language were translated by mental health professionals native in that language. We excluded quasirandomised controlled trials and N-of- 1 trials. We then restricted selection to studies that described patients with type 1 diabetes or insulin dependent diabetes. Studies combining type 1 and type 2 diabetes were included only if the study design was stratified by type of diabetes. For crossover trials only the first arm was included. Studies with multiple intervention arms provided only one study entry in any one analysis. The minimum number of sessions to define a psychological therapy was one. We coded type of therapy, number of sessions, duration of therapy, and format of delivery (defined as individual, group, or family (including multiple families)). We included trials where glycaemic control was a secondary outcome. Data were also extracted on sample size at baseline and at follow-up, clinical subgroups, children and adolescents versus adult, age, duration of diabetes, and duration of follow-up from baseline.

\section{Statistical analysis}

We used Stata 8 (StataCorp, College Station, TX, USA) with user contributed commands for meta-analyses (metan and metabias) for the analyses. ${ }^{20}$

The difference in mean change scores for glycaemic control (and for psychological functioning) from baseline to follow-up between treatment groups was standardised by dividing by the pooled standard deviation (SD) of the change scores within the group; if this was missing we calculated them from the SDs at baseline and at follow-up by assuming that the true SDs at baseline and follow-up were constant and the correlation between patient's baseline and follow-up values was moderately positive $(r=0.5)$. If there were several follow-up time periods, we used the glycated haemoglobin value recorded in the longest follow-up interval. We calculated the standard error of each study's standardised effect size estimate from the estimated effect and the study's group sizes according to a formula provided by Cooper and Hedges ${ }^{21}$ and used in metan.

The standardised effect sizes were pooled with a random effects model as we expected heterogeneity because of the variety of psychological treatments and settings. Random effects models produce wider confidence intervals and more conservative estimates than fixed effects models. The assumption of homogeneity of true effect sizes was assessed formally by applying Cochran's $Q$ test. ${ }^{22}$

The standardised effect sizes were also back transformed into percentage glycated haemoglobin by multiplying the standardised score with an estimate of the SD of the glycated haemoglobin change scores obtained as a weighted average of respective values across the studies that measured glycated haemoglobin.

We assessed whether conclusions were sensitive to restricting studies to subgroups that might modify the effects of therapy, such as family setting in children and adolescent studies or type of therapy in adult studies.

The presence of publication bias for the main experimental hypothesis of psychological intervention effects on glycaemic control was assessed informally by a funnel plot $^{23}$ and formally by its direct statistical analogue (Begg's adjusted rank correlation test). ${ }^{24}$

\section{Quality assessment}

We assessed studies for quality based on the three main quality criteria (selection bias, attrition bias, detection bias) specified by Schulz and by Jadad. ${ }^{25}$ Blinding of patients and the therapist was not assessed because psychological treatments do not allow them to be concealed from the therapist or patient. Studies were subdivided into the three categories: A (all quality criteria metlow risk of bias), $\mathrm{B}$ (one or more of the quality criteria partly met-moderate risk of bias), and $\mathrm{C}$ (one or more criteria not met-high risk of bias).

\section{Results}

\section{Systematic review}

The search strategy identified 3488 studies from which 121 full hard copies were selected for further extraction (see QUOROM flow chart on bmj.com). There was 95.4\% agreement about which abstracts to include for retrieval of full hard copies $(\kappa 0.70$, $95 \%$ confidence interval 0.68 to 0.73 ).

Sixteen randomised controlled trials of psychological interventions in children and adolescents (table A on bmj.com) and 13 in adults (table B on bmj.com) met the criteria for inclusion in the systematic review (a full list of references to included studies ${ }^{\text {w1-w34 }}$ is on bmj.com).

All but two trials ${ }^{\mathrm{w18}}{ }^{\mathrm{w} 20}$ in children and adolescents (table A on bmj.com) had sample sizes $<100$. The most common mode of delivery was multiple family or parent group. Adolescence was the most common specified clinical group. The mean duration of diabetes was 5.6 (SD 2.07) years and the mean duration of follow-up was 11.4 (7.0) months. One study was rated quality $\mathrm{A}^{\text {w19 }}$; eight studies were published after the CONSORT consensus. ${ }^{27}$ Seven studies examined cognitive behaviour 
therapy, ${ }^{\mathrm{w} 1-3} \mathrm{w} \mathrm{w}^{\mathrm{w} 9} \mathrm{w10} \mathrm{w14}$ six studies were of counselling, ${ }^{\mathrm{w} 5} \mathrm{w} 6 \mathrm{w} 8 \mathrm{w18-20}$ and three studies used family systems therapy. ${ }^{\mathrm{w} 4 \mathrm{w} 11 \mathrm{w} 16}$

All but two trials ${ }^{\text {w32 w33 }}$ in adults (table B on bmj.com) had sample sizes less than 100. Eight studies used either group format or a combination of group and individual formats. The clinical subgroups were suboptimal glycaemic control, ${ }^{\mathrm{w} 21 \mathrm{w} 25 \mathrm{w} 30 \mathrm{w} 33}$ new onset diabetes, ${ }^{\mathrm{w} 24}$ complications, ${ }^{\mathrm{w} 31}$ and obesity. ${ }^{\mathrm{w} 29}$ The mean duration of diabetes was 14.1 (SD 6.85) years and the mean duration of follow-up was 7.2 (SD 4.8) months. One study was rated as quality A..$^{\mathrm{w} 24}$ Most trials examined cognitive behaviour therapy, ${ }^{\text {w21-23 }}$ w26 w29-31w33 w34 two examined psychoanalytical techniques, ${ }^{\text {w24 w25 }}$ and two examined counselling. ${ }^{\text {w27 w32 }}$

\section{Meta-analysis of glycaemic control}

There were 10 studies in children and adolescents $(n=543$ participants) and 11 in adults $(\mathrm{n}=516$ participants) with data that could be pooled. With random effects meta-analyses, there was a small to moderate pooled estimate of the mean standardised effect sizes $(-0.35(95 \%$ confidence interval -0.66 to -0.04 , $\mathrm{P}=0.03$ ) combined across all studies in children, but this association was attenuated when we combined data across all studies in adults $(-0.17,-0.45$ to $0.10, \mathrm{P}=0.22)$ (fig 1$)$. The standardised effects translated into absolute reductions in glycated haemoglobin of $0.48 \%(0.05 \%$ to $0.91 \%)$ for children and adolescents and of $0.22 \%(-0.13 \%$ to $0.56 \%)$ for adults. Cochran's $Q$ test indicated heterogeneity $(\mathrm{P}=0.002$ for studies in child and adolescents and $\mathrm{P}=0.02$ in studies in adults).

For the sensitivity analysis, restriction to family therapies slightly increased the pooled standardised effect of therapy for

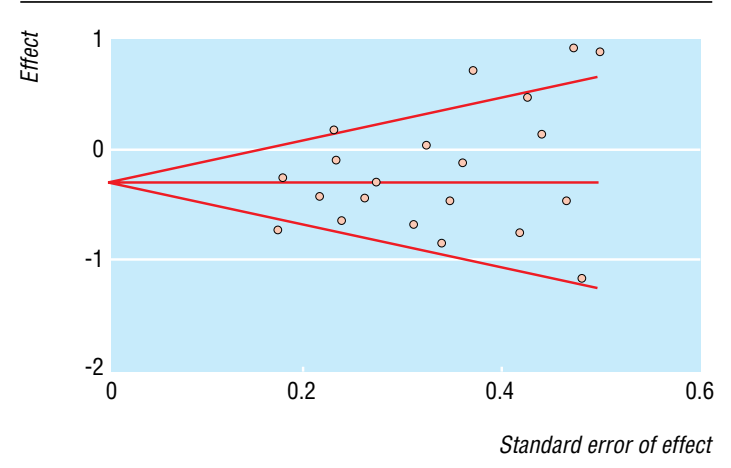

Fig 2 Publication bias in randomised controlled trials of psychological interventions in children, adolescents, and adults with type 1 diabetes (Begg's funnel plot with pseudo $95 \%$ confidence intervals)

children and adolescents $(-0.41,-0.79$ to $-0.03, \mathrm{P}=0.03)$. Restriction to group cognitive behaviour therapy further attenuated the pooled standardised effect size for adults $(0.02,-0.41$ to $0.44, \mathrm{P}=0.95$ ).

\section{Publication bias}

A funnel plot based on all 21 studies with glycaemic control measures (fig 2) did not indicate any publication bias nor did the formal test (Begg's adjusted rank correlation test, $\mathrm{P}=0.17$ ).

\section{Meta-analysis of psychological distress}

Fourteen studies used a continuous measure of psychological

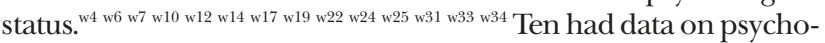

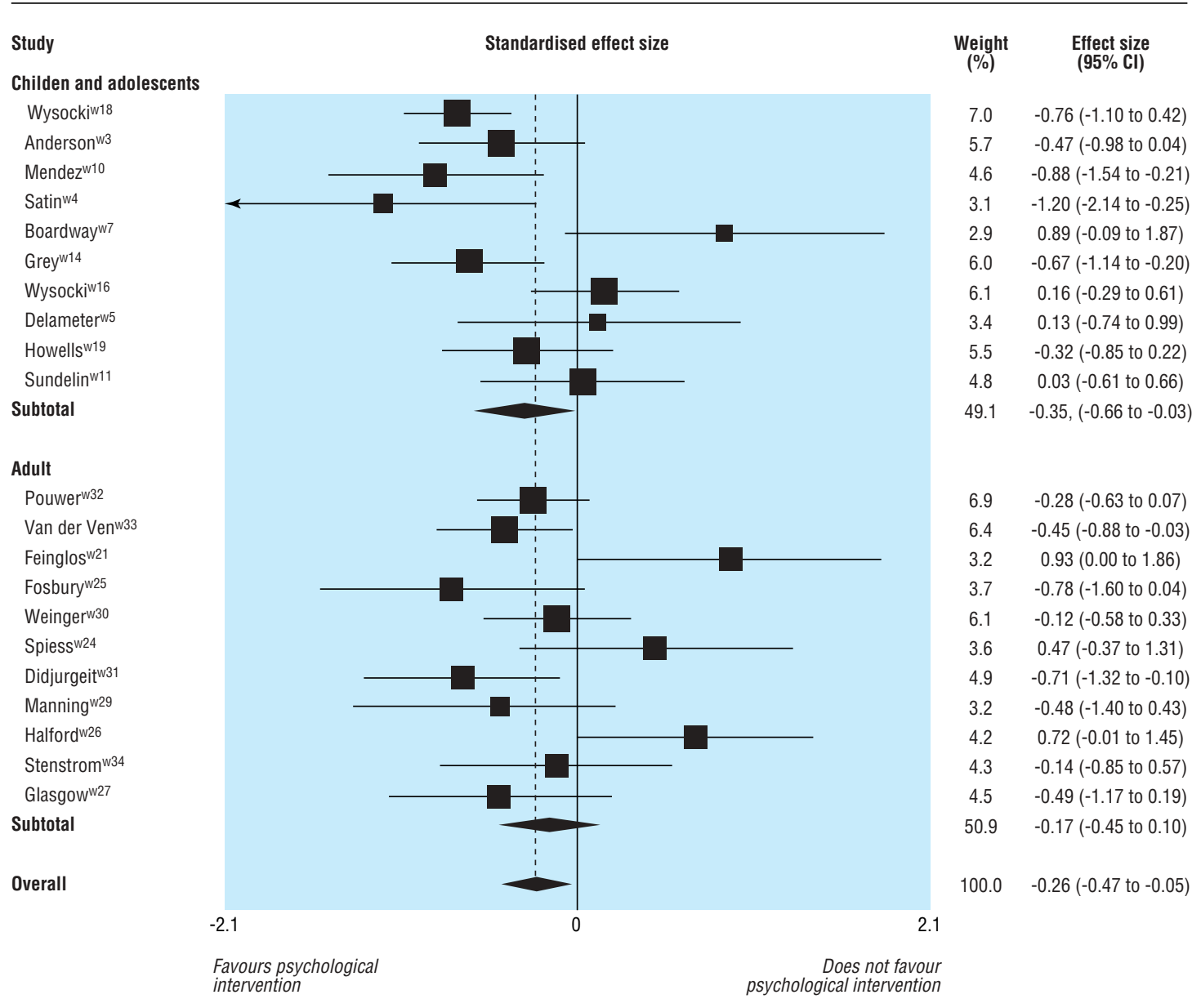

Fig 1 Standardised effects of psychological intervention on percentage of glycated haemoglobin in children and adolescents and in adults 
logical outcomes ( $\mathrm{n}=417$ participants) that could be pooled in a meta-analysis. Figure 3 shows that all selected studies reported an improvement in psychological distress.

With random effects meta-analyses the pooled estimate of the mean therapy effect on psychological distress for children and adolescents was moderate (standardised effect size -0.46 , -0.83 to $-0.10, \mathrm{P}=0.013)$. The effect was attenuated in adults (standardised effect size $-0.25,-0.51$ to $0.01, \mathrm{P}=0.059$ ). Cochran's $Q$ test did not find any evidence for heterogeneity in psychological distress effects (children and adolescents $\mathrm{P}=0.23$; adults $\mathrm{P}=0.74)$.

\section{Discussion}

In this systematic review of psychological interventions for improving diabetes control we found that psychological therapy was associated with a significant improvement in glycaemic control in the 10 studies in children and adolescents, with a pooled absolute reduction in glycated haemoglobin of $0.5 \%$. In the 11 studies in adults in the meta-analysis this association was smaller and not significant.

The methodological quality of most studies was moderate to poor. The estimated $0.5 \%$ reduction in glycated haemoglobin in children and adolescents was small but sufficient to reduce the risk of development and progression of diabetic microvascular complications. ${ }^{7}$ The success of psychological interventions in children may be explained by higher levels of psychological distress in children and their families; cohort studies have reported that family functioning is associated with glycaemic control. ${ }^{28}$ Alternatively parents of children with diabetes may be more responsive to psychological interventions than their offspring, as has been suggested in caregivers of adolescents with eating disorders. ${ }^{29}$ There were no studies using family therapy for adults to compare with the 10 for children and adolescents.

Most of the psychological interventions used variants of cognitive behaviour therapy. This is flexible, focused, and time limited and is increasingly being adopted as an intervention in management of chronic disease. ${ }^{11}$ No trial tested the effectiveness of motivational interviewing therapies, and only two studies used psychoanalytical therapies, ${ }^{\text {w24 }}{ }^{\text {w25 }}$ suggesting these are understudied.

The improvements in psychological functioning, especially in children and adolescents, highlight the important levels of psychological distress among people with diabetes, yet only one study targeted those with manifest psychological problems. ${ }^{\mathrm{w} 16}$ Our review found that group interventions may have potentially underestimated effect sizes in the sensitivity analysis. From our review, it is unclear whether individual therapies could be more effective in improving control. None of the studies reported taking into account preferences of patients in type or format of therapy.

\section{Conclusions}

We found weak evidence for the effectiveness of psychological treatments in improving glycaemic control in children and adolescents but not in adults. Psychological techniques applied in diabetes are still in their infancy. Future research should focus on improving methods to CONSORT standards, developing and refining theoretically based models for psychological interventions specific for diabetes, incorporating patients' preferences, and examining which types of therapies are effective for which subgroups of people with diabetes.

We thank Hugh McGuire (Institute of Psychiatry, London), who carried out the electronic searches; the Cochrane Metabolic and Endocrine Disorders Group, who peer reviewed the original protocol; Elvira Bramon Bosch (Institute of Psychiatry, London), who translated the Spanish papers; and Frank Snoek, Nicole van der Ven, and Frans Pouwer (Vrije University, Amsterdam, Netherlands), Margaret Grey (Yale University, Connecticut, USA), R T Jung (Ninewells Hospital and Medical School, Dundee), Ulf Stenström (University College of Health Sciences, Jönköping, Sweden), and Deborah Toobert (Oregon Research Institute, Oregon, USA), for giving us access to and permission to use their unpublished data for the meta-analyses and answering our queries.The protocol was peer reviewed and published in the Cochrane Database of Systematic Reviews (www.cochrane.org/reviews/en/topics/77.html).

Contributors: KW collected and extracted the data, contributed to the statistical analysis, and drafted the manuscript; SL carried out the statistical analysis and prepared the manuscript; IE analysed the psychological therapies and prepared the manuscript; KI developed the research hypotheses and protocol, collected and extracted the data, prepared the manuscript, and is guarantor.

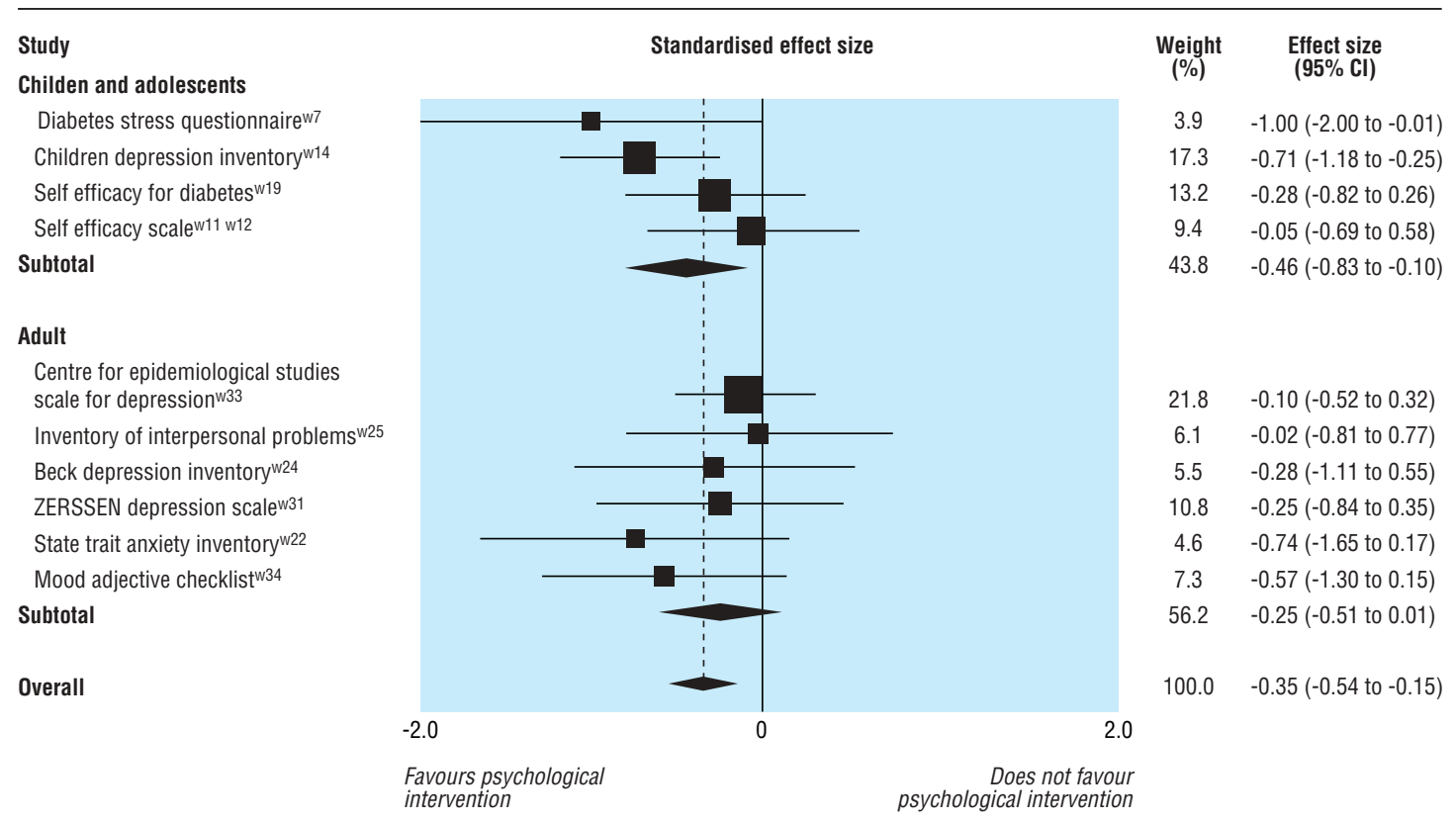

Fig 3 Standardised effects of psychological intervention on psychological distress for children and adolescents and adults 
Funding: None.

Competing interests: None declared.

Ethical approval: Not required.

1 Snoek F, Skinner T. Psychological counselling in problematic diabetes: does it help? Diabet Med 2002;19:265-73.

2 Lustman P, Anderson R, Freedland K, de Groot M, Carney R, Clouse R. Depression and poor glycaemic control. A meta-analytic review of the literature. Diabetes Care 2000;23:934-42

3 Anderson R, Freedland K, Clouse R, Lustman P. The prevalence of comorbid depression in adults with diabetes: a meta-analysis. Diabetes Care 2001;24:1069-78.

4 Peveler R, Bryden K, Neil A, Fairburn C, Mayou R, Dunger, et al. The relationship of disordered eating habits and attitudes to clinical outcomes in young adult females with type 1 diabetes. Diabetes Care 2005;28:84-8.

5 Cox D, Irvine A, Gonder-Frederick L, Nowacek G, Butterfield J. Fear of hypoglycaemia: quantification, validation and utilization. Diabetes Care 1987;10:617-21.

6 Gold AE, Deary IJ, Frier BM. Hypoglycaemia and non-cognitive aspects of psychological function in insulin-dependent (type 1) diabetes mellitus (IDDM). Diabet Med 1997;14:111-8.

7 Diabetes Control and Complications Trial Research Group. The effect of intensive treatment of diabetes on the development and progression of long-term complications in insulin-dependent diabetes mellitus. N Engl J Med 1993;329:977-86.

8 National Institute for Clinical Excellence. Type 1 diabetes: diagnosis and management of type 1 diabetes in children, young people and adults. London: National Institute for Health and Clinical Excellence, 2004.

9 Griffin S, Kinmouth AL, Skinner C, Kelly J. Educational and psychosocial interventions for adults with diabetes: report to the British Diabetic Association. London: British Diabetic Association, 1998 .

\section{What is already known on this topic}

Suboptimal glycaemic control in people with type 1 diabetes is associated with increased psychological distress, morbidity, and mortality

Individual randomised controlled trials suggest that psychological treatments may help to improve glycaemic control

\section{What this study adds}

There is weak evidence that psychological treatments could improve glycaemic control in children and adolescents and no evidence of an effect in adults

Cognitive behaviour therapy techniques were more common than counselling or psychoanalytical techniques

Family therapies may be more effective than individual therapies for children and adolescents with diabetes
10 Hampson S, Skinner T, Hart J, Storey L, Gage H, Foxcroft D, et al. Effects of educational and psychosocial interventions for adolescents with diabetes mellitus: a systematic review. Health Technol Assess 2001;5:1-79.

11 Hodes M, Moorey S. Psychological treatment in disease and illness. London: Gaskell, 1993.

12 Churchill R, Hunot V, Corney R, Knapp M, McGuire H, Tylee A, et al. A systematic review of controlled trials of the effectiveness and cost-effectiveness of brief psychological treatments for depression. Health Technol Assess 2001;5:1-173.

13 Miller W, Sovereign G, Kree B. Motivational interviewing with problem drinkers: II, The drinkers check-up as a preventative intervention. Behav Psychother 1988;16:251-68.

14 Beck A. Cognitive therapy and the emotional disorders. New York: Meridian, 1976.

15 Malan D. A study of brief psychotherapy. London: Plenum Press, 1963

16 Minuchin S. Families and family therapy. London: Routledge, 1991.

17 Moorey S, Williams R. Behavioural and cognitive therapies. In: Murray R, Hill P, McGuffin P, eds. The essentials of postgraduate psychiatry. Cambridge: Cambridge University Press, 1997.

18 Miller W, Rollnick S. Motivational interviewing: preparing people to change addictive behaviour. New York: Guildford Press, 1991.

19 Fleiss J. Statistical methods for rates and proportions. 2nd ed. New York: Wiley, 1981.

20 Bradburn M, Deeks J, Altman D. sbe24: metan-an alternative meta-analysis command. Stata Tech Bull 1998;440:4-15.

21 Cooper H, Hedges L. The handbook of research synthesis. New York: Russell Sage Foundation, 1994.

22 Cochran W. The comparison of percentages in matched samples. Biometrika 1950;37:256-66.

23 Egger M, Smith G, Schneider M, Minder C. Bias in meta-analysis detected by a simple graphical test. $B M J$ 1997;315:629-34

24 Begg C, Mazumdar M. Operating characteristics of a rank correlation test for publication bias. Biometrics 1994:50:1088-101.

25 Schulz K, Chalmers I, Hayes R, Altman D. Empirical evidence for bias: dimensions of methodological quality associated with estimates of treatment effects in controlled trials. JAMA 1995;273:408-12.

26 Jadad A, Moore R, Carroll D, Jenkinson C, Reynolds J, Gavaghan D, et al. Assessing the quality of reports of randomized clinical trials: is blinding necessary? Control Clin Trials $1996 ; 17: 1-12$.

27 Moher D, Schulz K, Altman D, for the CONSORT Group. The CONSORT statement: revised recommendations for improving the quality of reports of parallel-group randomised trials. Lancet 2001;357:1191-4.

28 Jacobson AM, Hauser ST, Lavori P, Willett JB, Cole CF, Worlsdorf JI, et al. Family environment and glycaemic control: a four year prospective study of children and adolescents with insulin dependent diabetes mellitus. Psychosom Med 1994;56:401-9.

29 Whitney J, Murray J, Gavan K, Todd G, Whitaker W, Treasure J. Experience of caring for someone with anorexia nervosa: qualitative study. Br J Psych 2005;187:444-9.

(Accepted 13 April 2006)

doi $10.1136 /$ bmj.38874.652569.55

Department of Psychological Medicine, Institute of Psychiatry, King's College London, London SE5 9RJ

Kirsty Winkley research nurse

Khalida Ismail senior lecturer in liaison psychiatry

Department of Biostatistics, Institute of Psychiatry, King's College London

Sabine Landau senior lecturer in biostatistics

Department of Psychology, Institute of Psychiatry, King's College London

Ivan Eisler reader in family therapy

Correspondence to: K Ismail khalida.ismail@iop.kcl.ac.uk 